Ion energy and angular distributions onto polymer surfaces delivered by dielectric barrier discharge filaments in air: I. Flat surfaces

This article has been downloaded from IOPscience. Please scroll down to see the full text article.

2011 Plasma Sources Sci. Technol. 20035017

(http://iopscience.iop.org/0963-0252/20/3/035017)

View the table of contents for this issue, or go to the journal homepage for more

Download details:

IP Address: 141.211.173.82

The article was downloaded on 06/04/2012 at 16:27

Please note that terms and conditions apply. 


\title{
Ion energy and angular distributions onto polymer surfaces delivered by dielectric barrier discharge filaments in air: I. Flat surfaces
}

\author{
Natalia Yu Babaeva and Mark J Kushner ${ }^{1}$ \\ University of Michigan, Department of Electrical Engineering and Computer Science 1301 Beal Ave, \\ Ann Arbor, MI 48109, USA \\ E-mail: nbabaeva@umich.edu and mjkush@umich.edu
}

Received 3 January 2011, in final form 22 March 2011

Published 28 April 2011

Online at stacks.iop.org/PSST/20/035017

\begin{abstract}
In atmospheric pressure discharges, ion energies are typically thought to be thermal with values dominantly $<1 \mathrm{eV}$. In the heads of filaments in dielectric barrier discharges (DBDs), electric fields can exceed $200 \mathrm{kV} \mathrm{cm}^{-1}$ when the filament is far from a surface. As the filament approaches and intersects a dielectric surface, much of the applied potential is compressed into the voltage drop across the head of the filament due to the high conductivity of the trailing plasma channel. When the filament strikes the surface, this voltage is transferred to the resulting sheath and into the material of the surface. The degree of electric field compression depends on the dielectric constant $\varepsilon / \varepsilon_{0}$ of the surface. Upon intersection of the filament with the surface, the electric fields in the resulting sheath can exceed $400-800 \mathrm{kV} \mathrm{cm}^{-1}$, with larger values corresponding to larger $\varepsilon / \varepsilon_{0}$. When accelerated in these fields, ions can gain energies across their mean free path $(0.5-1 \mu \mathrm{m})$ up to $20 \mathrm{eV}$ for dielectrics with low $\varepsilon / \varepsilon_{0}$ and up to $150 \mathrm{eV}$ for dielectrics with high $\varepsilon / \varepsilon_{0}$, albeit only for the duration of the intersection of the streamer with the surface of a few ns. In this paper we report on results from a computational investigation of the ion energy and angular distributions (IEADs) incident on dielectric flat surfaces resulting from the intersection of DBD filaments sustained in atmospheric pressure air. We describe the transient and spatially dependent IEADs as the filament spreads across the polymer.
\end{abstract}

(Some figures in this article are in colour only in the electronic version)

\section{Introduction}

Plasmas generated at atmospheric pressure, often in a dielectric barrier discharge (DBD) configuration, are frequently used to functionalize the surfaces of polymers or biomedical devices by creating new surface-resident chemical groups [1-3]. Surfaces in contact with DBDs are simultaneously exposed to chemically reactive neutral radicals produced by the discharge, UV radiation and ion bombardment. Hydrocarbon polymer films modified by DBDs sustained in air often show an increase in surface energy due to the introduction of oxygen-containing

\footnotetext{
${ }^{1}$ Author to whom any correspondence should be addressed.
}

groups on the surface and to some degree due to the etching of the surface. While there have been extensive studies on radicals and UV radiation from atmospheric pressure plasmas (APPs) impinging on polymer surfaces [4-7], little is known on the range of ion energies incident onto these surfaces that can be produced by APPs.

The importance of the ion energy and angular distributions (IEADs) produced in APPs onto surfaces goes beyond polymer processing. The therapeutic effects of APPs in direct contact with living tissue are also partially attributed to energetic ions impinging onto wounds and tissue surfaces [8,9]. Although ions probably do not penetrate beyond the top layers of cells, their energetic fluxes are non-negligible. Typical plasma 
sources used for treatment of tissue are multi-filamentary DBDs operating at up to tens of $\mathrm{kHz}$ in a floating electrode configuration where one electrode is the tissue being treated. Individual filaments are a few ns in duration and are statistically distributed between the electrodes.

In APPs, charge separation in the head of a filament can produce electric fields up to $150-200 \mathrm{kV} \mathrm{cm}^{-1}$ (or $E / N$ (electric field/gas density) $=600-800 \mathrm{Td}, 1 \mathrm{Td}=$ $10^{-17} \mathrm{Vcm}^{2}$ ) [10]. These electric fields sustain the electron avalanche that propagates as a filament a few hundred micrometers in diameter. The majority of the applied voltage drops across the head of the filament and across the non-ionized gas ahead of the filament-relatively little voltage drops across the plasma channel trailing behind the filament due to its higher conductivity. When a filament strikes a surface, thereby bridging the gap with a conductive channel, the majority of the applied voltage is momentarily transferred to the sheath at the dielectric surface. The degree of penetration of the applied potential into the dielectric depends on the dielectric constant $\varepsilon / \varepsilon_{0}$ of the material and the degree of surface charging. At the surface of the dielectric in a DBD, with plasma densities of $2 \times 10^{14} \mathrm{~cm}^{-3}$ to $10^{15} \mathrm{~cm}^{-3}$ and potential drops of $2-10 \mathrm{kV}$ in the sheath, the sheath thickness is a few tens of $\mu \mathrm{m}$. This results in electric fields of the order of a few hundreds of $\mathrm{kV} \mathrm{cm}^{-1}$ lasting for many ns. With mean free paths below $1 \mu \mathrm{m}$ for ions at atmospheric pressure, one might expect ion energies of up to tens of $\mathrm{eV}$ incident onto the surface produced by acceleration in the sheath.

In this paper, we report on results from a computational investigation of the IEADs produced by DBD filaments which are incident onto flat polymer surfaces backed by a ground plane. We found that ion energies of many tens of $\mathrm{eV}$ may be transiently incident onto the flat polymer surface produced by electric fields in the sheaths of many hundreds of $\mathrm{kV} \mathrm{cm}^{-1}$. IEADs incident onto small electrically floating polymer surfaces, such as a dust particle, drug delivery bead or bacteria, are discussed in the companion paper, part II [11].

The description of the model is given in section 2 . The propagation of filaments in a DBD and the resulting ion energies onto flat polymer surfaces with low and high $\varepsilon / \varepsilon_{0}$, and as a function of voltage, are discussed in section 3 . Our concluding remarks are in section 4.

\section{Description of the model}

The physical processes and algorithms used in the model are discussed in detail in [12] and so will be only briefly reviewed here. The model, nonPDPSIM, is a multi-fluid hydrodynamics simulation in which transport equations for all charged and neutral species and Poisson's equation are integrated as a function of time. Updates of the charged particle densities and electric potential are followed by an implicit update of the electron temperature, $T_{\mathrm{e}}$, by solving the electron energy conservation equation. The electron transport coefficients and rate coefficients for bulk electrons as a function of average electron energy $\left(\langle\varepsilon\rangle=\frac{3}{2} k T_{\mathrm{e}}\right)$ are obtained by solving Boltzmann's equation for the electron energy distribution.

Poisson's equation was solved throughout the entire computational domain (except in metals where the potential is specified as a boundary condition). Continuity equations for gas phase charged and neutral particles are only solved in the plasma region. Conservation equations for surface and volume charge are solved on and inside all non-metallic materials.

Our approach to photoionization is based on line-of-sight propagation of UV ionizing radiation generated by high lying excited states that are produced largely in the high $E / N$ regions in the avalanche front. The UV radiation is absorbed (without producing ionization) over a specified mean free path which determines its extent beyond its origin. We assumed that ionization occurs by absorption by $\mathrm{O}_{2}$ molecules of photons emitted by $\mathrm{N}_{2}\left(b^{1} \Pi\right)$ and $\mathrm{N}_{2}\left(b^{1} \Sigma\right)$ in the wavelength range $98-102 \mathrm{~nm}$. The non-ionizing absorption cross section was approximated as $2 \times 10^{-17} \mathrm{~cm}^{-2}$ while that for the ionizing cross section is $10^{-18} \mathrm{~cm}^{2}$. In the absence of inhomogeneities, the precise value of these cross sections does not significantly affect the calculation other than in the speed of propagation of the streamer provided that the ionizing cross section is large enough to prevent the streamer from stalling.

An unstructured mesh having a dynamic range of $>1000$ is used to enable both the DBD reactor scale and the sheath at the surface of the flat dielectric (or floating particle) to be resolved in a single mesh. The unstructured numerical mesh has triangular elements with refinement regions. The meshes consisted of approximately 5000-7000 nodes, of which about 4000-6000 are in the plasma region. The mesh resolution, as shown in figure $1(b)$, is $1-4 \mu \mathrm{m}$ near the anode and cathode (or dielectrics covering those electrodes).

IEADs to surfaces are computed using the Plasma Chemistry Monte Carlo Module (PCMCM), as described in [13]. The PCMCM is executed on a Cartesian mesh that overlays a subset of the computational domain. Pseudoparticles representing ions are launched from sites within the PCMCM mesh with weightings proportional to their rate of generation by electron impact and heavy particle reactions. Pseudo-particles are also launched from the boundaries of the PCMCM mesh in proportion to the entering fluxes of ions. Monte Carlo techniques are used to advance their trajectories in time varying electric fields while accounting for elastic and inelastic collisions. The energy and angles of particles as they strike surfaces are recorded over a predetermined period of time to provide IEADs as a function of location on the polymer surface and time. Electric potentials as a function of position computed on the unstructured mesh are interpolated onto the rectilinear structured mesh that overlays the unstructured mesh to enable the Monte Carlo algorithms to more rapidly execute. As the filament evolves, snapshots of electric fields are transferred to the PCMCM to produce a time sequence of the IEADs to the surface. The time intervals between export of the electric fields to the PCMCM and the mesh resolution were extensively parametrized to ensure that the IEADs were not sensitive to these values. In the end, the typical resolution for structured PCMCM meshes was $0.4-1 \mu \mathrm{m}$.

The gas mixture used in the simulations is atmospheric pressure dry air $\mathrm{N}_{2} / \mathrm{O}_{2}=80 / 20$ at $300 \mathrm{~K}$. We used a reduced 


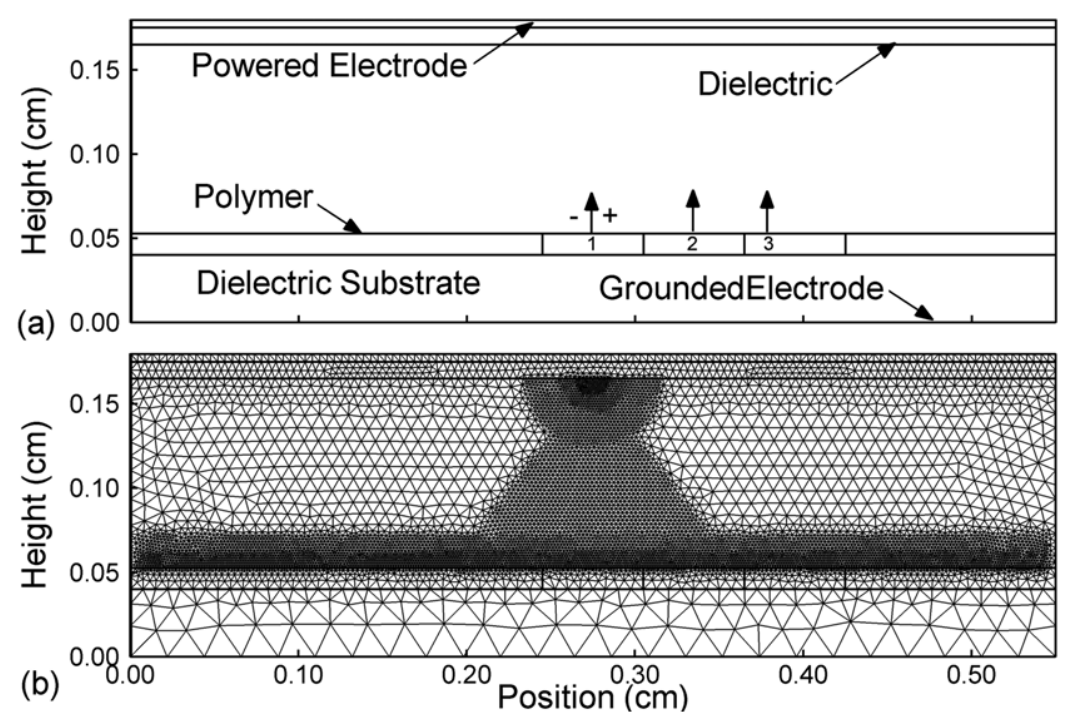

Figure 1. Geometry used for simulation of a single DBD filament and IEADs incident onto a flat polymer surface. (a) Total computational domain. The top powered electrode is covered by dielectric $\left(\varepsilon / \varepsilon_{0}=3\right)$ with thickness of $100 \mu \mathrm{m}$. The bottom grounded electrode is covered with $400 \mu \mathrm{m}$ thick dielectric $\left(\varepsilon / \varepsilon_{0}=2.2,16\right.$ or 80$)$. A thin polymer film $\left(\varepsilon / \varepsilon_{0}=2.2,127 \mu \mathrm{m}\right.$ thick $)$ is placed on the bottom dielectric. Sites where IEADs are collected are noted. $(b)$ Total computational domain showing unstructured mesh used to resolve filament structures and sheath regions.

reaction mechanism containing a subset of the reactions described in [12] to limit the magnitude of the calculation. We have confirmed that for the duration of the pulses examined in this work, the reduced reaction mechanism provides essentially the same results as the full reaction mechanism. The reduced reaction mechanism includes $\mathrm{N}_{2}, \mathrm{~N}_{2}^{*}, \mathrm{~N}_{2}^{* *}, \mathrm{~N}_{2}^{+}, \mathrm{O}_{2}$, $\mathrm{O}_{2}^{+}$and electrons. The states $\mathrm{N}_{2}^{*}$ and $\mathrm{N}_{2}^{* *}$ are nominally $\mathrm{N}_{2}\left(A^{3} \Sigma\right)$ and $\mathrm{N}_{2}\left(b^{1} \Pi, b^{1} \Sigma\right)$ though the latter is treated as a lumped state including transitions higher than $\mathrm{N}_{2}\left(A^{3} \Sigma\right)$. To initiate positive filaments, a small spot of seed-charges consisting of electrons and $\mathrm{N}_{2}^{+}$with peak density of $10^{9} \mathrm{~cm}^{-3}$ (diameter of $50 \mu \mathrm{m}$ in the horizontal direction and $100 \mu \mathrm{m}$ in the vertical direction) was placed at the center of the top dielectric shown in figure $1(a)$. There was otherwise no initial plasma density elsewhere in the computational domain.

The geometry for investigation of IEADs incident onto flat polymers is shown in figure 1 . A positive filament is sustained in the $1.1 \mathrm{~mm}$ gap between top dielectric and the polymer film. The powered electrode is biased to 15 or $20 \mathrm{kV}$. The top powered electrode is covered by a dielectric $\left(\varepsilon / \varepsilon_{0}=3\right)$ with thickness of $100 \mu \mathrm{m}$. The bottom grounded electrode is covered with a thicker $400 \mu \mathrm{m}$ dielectric whose dielectric constant was varied $\left(\varepsilon / \varepsilon_{0}=2.2,16\right.$ or 80$)$. A thinner polymer film with a dielectric constant $\varepsilon / \varepsilon_{0}=2.2$ and thickness of $127 \mu \mathrm{m}$ is placed on the bottom dielectric (as, for example, in the experiments described in [6]). IEADs will be identified as being incident onto sites labeled 1,2 and 3 as shown in figure 1 . Site 1 is directly under the filament as it strikes the surface. Sites 2 and 3 are at progressively larger distances from the intersection point (600 and $1200 \mu \mathrm{m}$ respectively). Ions arriving to the right of the normal vectors shown in figure $1(a)$ have positive angles while ions arriving to the left of the normal vector have negative angles.

\section{IEADs onto flat dielectric surfaces: low and high dielectric constants}

\subsection{Low dielectric constant}

The plasma in DBDs typically consists of filaments having area densities of tens to hundreds per $\mathrm{cm}^{2}$. These filaments treat surfaces uniformly due to the spreading of the discharge along the surface, the spatial averaging of many discharges and the diffusion of gas phase radicals. DBD filaments or streamers are narrow conductive plasma channels that are initiated near the anode for positive polarity and the cathode for negative, and propagate toward the opposite electrode that is usually covered by dielectric. The discharge develops in two phases. There is first the formation and propagation of an ionizing wave or filament across the discharge gap. Then, following intersection of the filament with and charging of the dielectric, a surface streamer is formed on the dielectric. When the discharge propagates along the surface, it is more appropriate to call it surface discharge or surface streamer.

The densities of oxygen ions during propagation of a filament as it crosses the inter-electrode gap and intersects the polymer having $\varepsilon / \varepsilon_{0}=2.2$ are shown in figure 2 for an applied voltage of $15 \mathrm{kV}$. Oxygen ion densities in the streamer channel reach $2.6 \times 10^{14} \mathrm{~cm}^{-3}$. Upon intersection with the polymer, the filament charges the surface, producing lateral electric fields which result in the spreading of the filament over the surface. The surface streamer, having an initial width of $700 \mu \mathrm{m}$ prior to striking the surface, spreads to a width of $5 \mathrm{~mm}$ in $7 \mathrm{~ns}$ for a speed of $7 \times 10^{7} \mathrm{~cm} \mathrm{~s}^{-1}$. The surface streamer is about $200 \mu \mathrm{m}$ in height above the dielectric. Similar spreading of the surface streamer was also shown in the modeling results of Papageorghiou et al [7]. In a multi-filament DBD, the intersection of adjacent surface streamers likely prevents the excessive spreading of plasma on the dielectric resulting in 


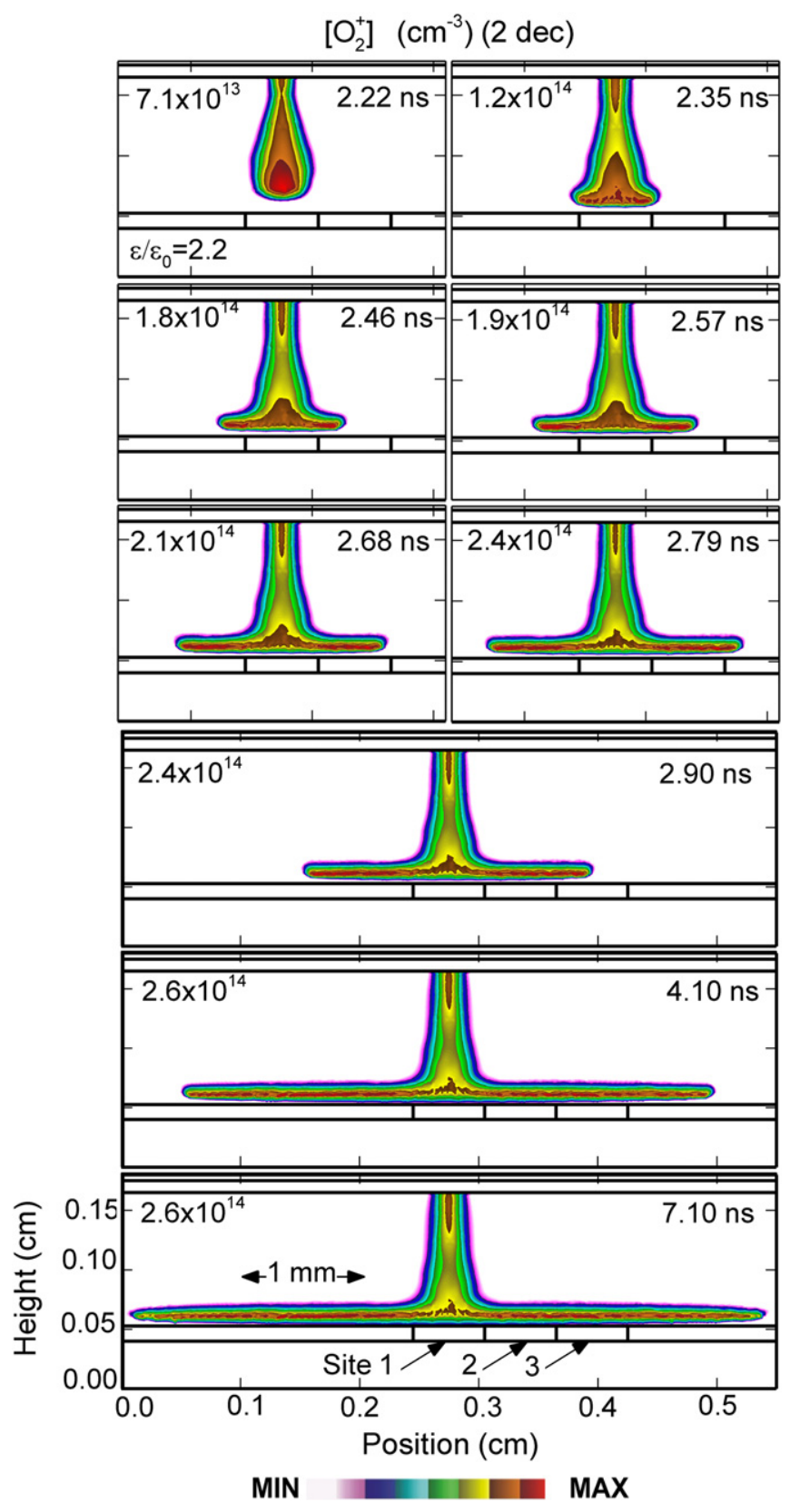

Figure 2. Density of oxygen ions as the filament approaches and spreads on the surface as a function of time (noted in each frame). The polymer and substrate have $\varepsilon / \varepsilon_{0}=2.2$ and the applied voltage $15 \mathrm{kV}$. The top six frames show the central part of the computational domain. The bottom three frames show the full computational region. The time sequence corresponds to that shown in figure 5 for IEADs.

self-organized plasma spots $[14,15]$. In their modeling of atmospheric pressure streamers in air intersecting a dielectric surface (Pyrex, $\varepsilon / \varepsilon_{0}=4.6$ ), Celestin et al [16] found similar spreading rates of $5 \times 10^{7} \mathrm{~cm} \mathrm{~s}^{-1}$.

In the head of the filament when the streamer is far from the surface electric fields reach up to $200 \mathrm{kV} \mathrm{cm}^{-1}$ (800 Td). The trailing conductive channel behind the streamer head drops a small voltage in comparison with that sustained across the head of the streamer. (Only $4 \mathrm{kV}$ is dropped across the streamer channel when the streamer intersects the polymer.) As a result, as the streamer approaches the surface, much of the electrode

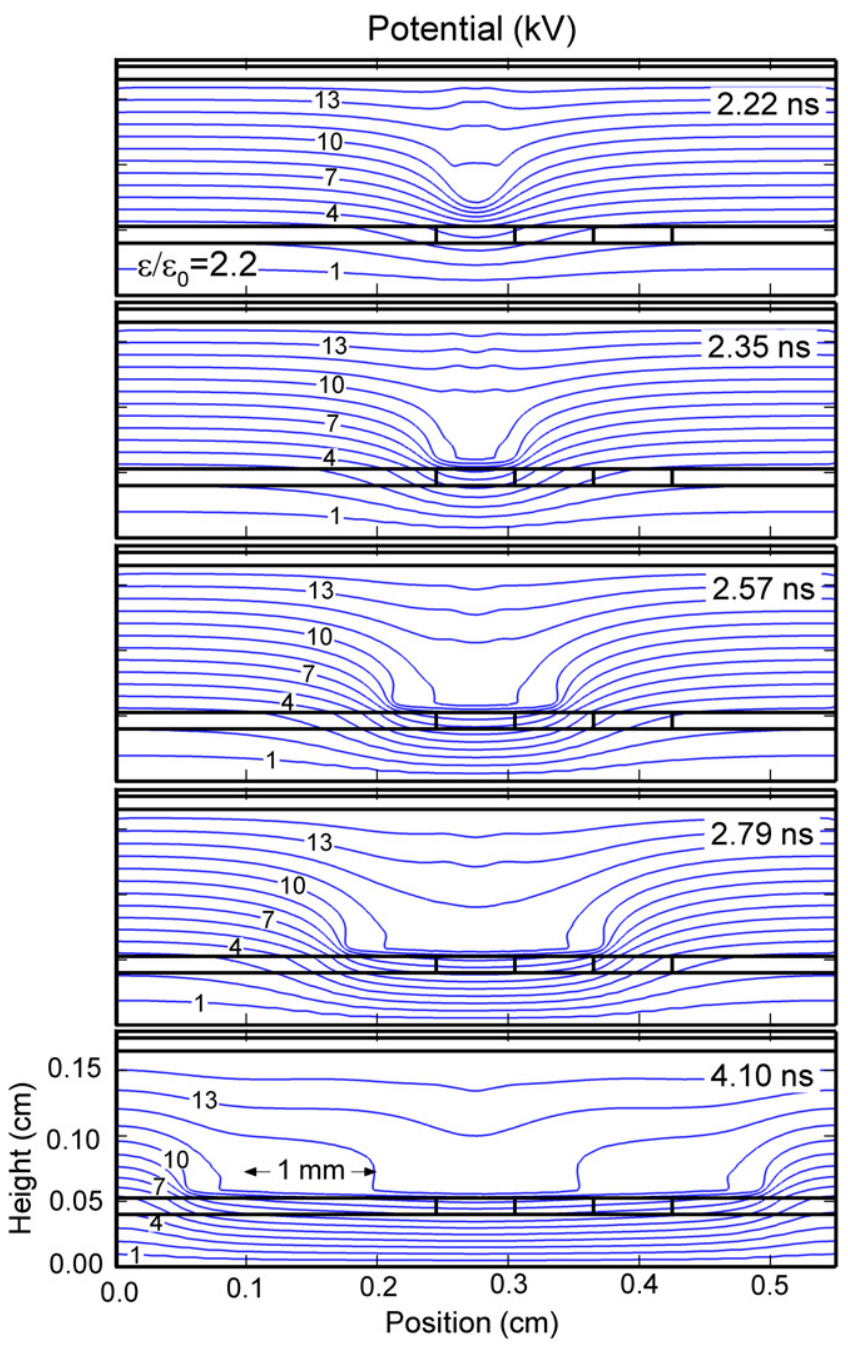

Figure 3. Potential contours as a function of time (subset of frames shown in figure 2) for the polymer and substrate having $\varepsilon / \varepsilon_{0}=2.2$ and applied voltage $15 \mathrm{kV}$. As the filament crosses the gap and strikes the polymer, potential is compressed nearer the polymer. For a polymer and substrate with low dielectric constant $\left(\varepsilon / \varepsilon_{0}=2.2\right)$ there is substantial penetration of potential inside the dielectrics. Contours are plotted every $1 \mathrm{kV}$.

potential is compressed between the head of the filament and the surface as shown in figure 3. When the filament strikes the surface, much of this voltage is then transferred to the resulting sheath at the surface. For polymers and substrates having a low dielectric constant $\left(\varepsilon / \varepsilon_{0}=2.2\right)$ there is substantial penetration of potential inside the dielectrics. Upon intersection of the streamer with the surface, the electric field in the sheath exceeds $400 \mathrm{kV} \mathrm{cm}^{-1}(1600 \mathrm{Td})$, as shown in figure 4(a), for a sheath thickness of $50 \mu \mathrm{m}$. As this is a positive streamer, the excess charge density at the periphery of the streamer (both in the gas phase and on the surface) is positive, as shown in figure $4(b)$. These high-voltage sheaths are transient due to the transient nature of the filament as the total filament duration is a few ns.

As the streamer spreads along the surface, large electric fields are also translated laterally along the surface to enable propagation of the streamer parallel to the surface. As the streamer passes over and charges the surface, the residual 


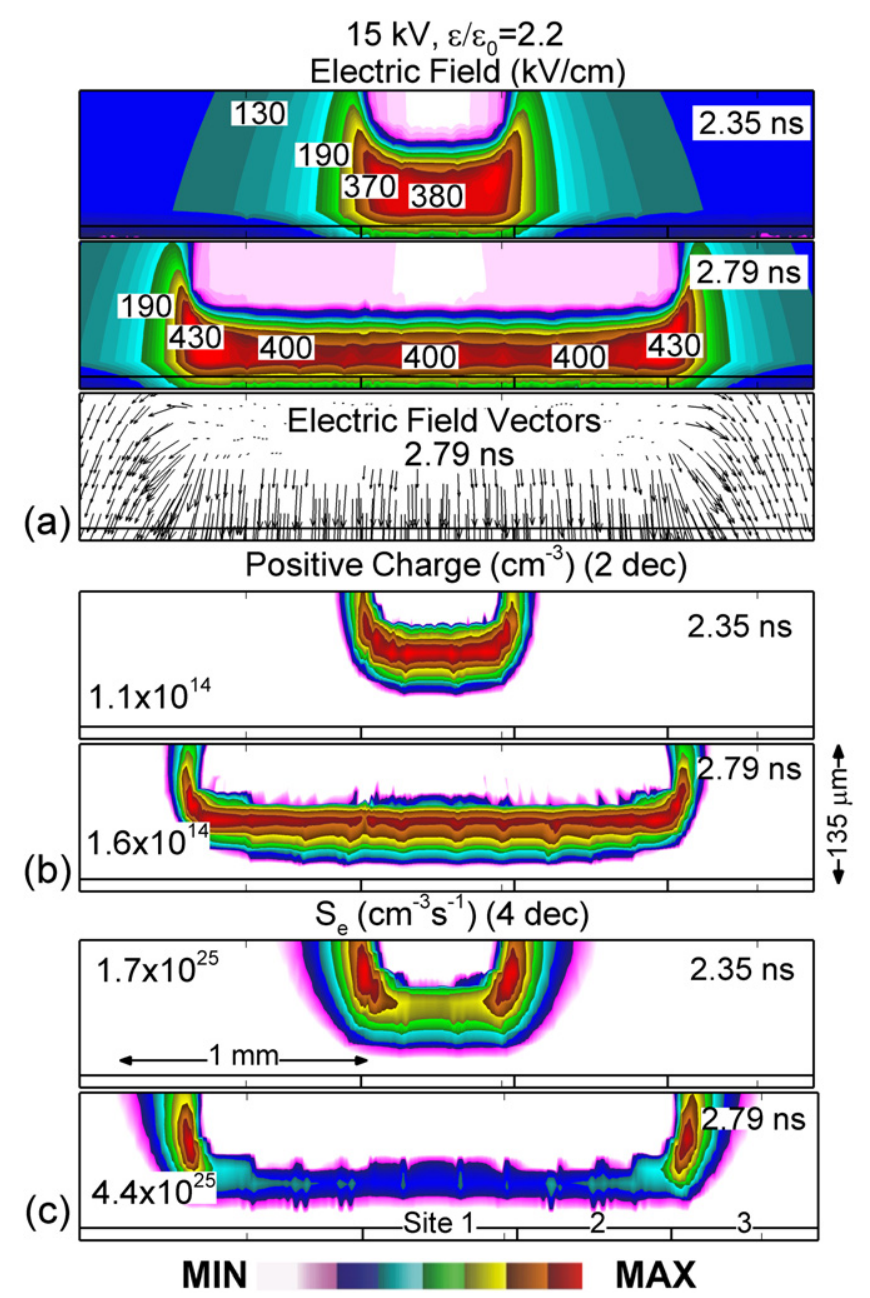

Figure 4. Plasma characteristics when the filament strikes and spreads on the polymer (subset of frames shown in figure 1). (a) Electric field and electric field vectors, $(b)$ positive space charge and (c) electron impact ionization sources. The polymer and substrate have $\varepsilon / \varepsilon_{0}=2.2$ and the applied voltage is $15 \mathrm{kV}$. By $2.79 \mathrm{~ns}$ the electric field reaches $400 \mathrm{kV} \mathrm{cm}^{-1}$ in the transient sheath. Electric field vectors are directed perpendicular to the polymer surface, except at the tips of the surface streamer where the vectors have significant parallel components. Note the high electron impact source at the streamer tip. The vertical to horizontal scale ratio is 4 .

electric field behind the streamer head is directed perpendicular to the polymer surface, as shown in figure $4(a)$. At the tip of the streamer the electric field has a significant component parallel to the surface. For example, at $2.8 \mathrm{~ns}$, the vertical component of the electric field behind the streamer head is $-415 \mathrm{kV} \mathrm{cm}^{-1}$ (around $1600 \mathrm{Td}$ ), whereas at the tip of the streamer the parallel component of the electric field is $140 \mathrm{kV} \mathrm{cm}^{-1}(560 \mathrm{Td})$. The electron temperatures corresponding to these electric fields in the leading edge of the streamer are 4.5-7.5 eV. Corresponding electron impact ionization sources at the leading edge of the streamer exceed $4.4 \times 10^{25} \mathrm{~cm}^{-3} \mathrm{~s}^{-1}$.

Electric fields were measured by Starikovskaia et al [17] by time-resolved spectroscopy of emission from $\mathrm{N}_{2}$ in nanosecond surface discharges sustained in air at pressures of 200 Torr to $1 \mathrm{~atm}$. Voltage pulses were $25 \mathrm{~ns}$ in duration and $20 \mathrm{kV}$ amplitude. Typical electric fields were $200 \mathrm{kV} \mathrm{cm}^{-1}$ $(800 \mathrm{Td})$ in a $1 \mathrm{~atm}$ discharge for negative polarity, and up to $350 \mathrm{kV} \mathrm{cm}^{-1}(1400 \mathrm{Td})$ for positive polarity. These values are commensurate with those predicted here for the surface discharge phase of the streamer propagation. In their modeling of streamers in air intersecting and spreading on Pyrex, Celestin et al [16] found electric fields in excess of $200 \mathrm{kV} \mathrm{cm}^{-1}$ in the head of streamer as it approached the surface, and in excess of $300 \mathrm{kV} \mathrm{cm}^{-1}$ at the head of surface discharge as it spread on the surface.

IEADs incident on the three sites along the polymer surface as a function of time are shown in figure 5 corresponding to the filament positions shown in figure 2. Each frame integrates the ion fluxes striking the polymer in $0.1 \mathrm{~ns}$. Typical ion mean free paths at atmospheric pressure are of the order of $0.5-1 \mu \mathrm{m}$. Given the magnitude of the electric fields at the surface of the polymer (hundreds of $\mathrm{kV} \mathrm{cm}^{-1}$ ), even with these short mean free paths ions can gain energies across their mean free path up to $30 \mathrm{eV}$.

Electron and ions are produced in front of the streamer head by photoionization. When the streamer is far from the surface $(2.22 \mathrm{~ns})$, small fluxes of $\mathrm{O}_{2}^{+}$to the substrate, $10^{15} \mathrm{~cm}^{-2} \mathrm{~s}^{-1}$, are produced by this mechanism. As these ions are produced in the vacuum electric fields, unenhanced by space charge, they largely drift to the polymer surface and arrive with epithermal energies with broad angular distributions. The ions are not strictly thermal (meaning gas temperature) since the vacuum electric fields at these times are already around $20-30 \mathrm{kV} \mathrm{cm}^{-1}$, which for a mean free path of $\lambda<1 \mu \mathrm{m}$, could result in average energies of ions striking the surface of about $1 \mathrm{eV}$. The effective energy [18] $\varepsilon_{\text {eff }}=\frac{3}{2} k_{\mathrm{B}} T_{\text {ion }}+\frac{1}{2} M v_{\mathrm{d}}^{2}$ ( $T_{\text {ion }}$ is the ion temperature, $v_{\mathrm{d}}$ is the drift velocity) captures the above thermal energy due to drift in large electric fields. For $50 \mathrm{kV} \mathrm{cm}^{-1}$ (200 Td), typical ion mobility of $3-4 \mathrm{~cm}^{2} \mathrm{~V}^{-1} \mathrm{~s}^{-1}$ and $T_{\text {ion }} \sim 0.03 \mathrm{eV}, \varepsilon_{\text {eff }}$ is approximately $1 \mathrm{eV}$. Since the vacuum fields are largest directly under the anode at site 1 , the IEADs at this time extend somewhat greater in energy at site 1 .

As the streamer continues to approach the surface $(2.35 \mathrm{~ns})$, the vacuum electric fields ahead of the streamer increase due to the voltage compression caused by the conductive streamer channel. This occurs at a time that the ion fluxes increase to $10^{16}-10^{17} \mathrm{~cm}^{-2} \mathrm{~s}^{-1}$ due to higher rates of photoionization produced by the proximity of the streamer-and the beginning of conduction currents. Ion energies increase to as high as $10 \mathrm{eV}$. When the streamer strikes the surface $(2.46 \mathrm{~ns})$, the electric field in the head of the streamer is transferred to the sheath at the surface of the polymer producing electric fields up to $400 \mathrm{kV} \mathrm{cm}^{-1}$. The sheath width is approximately $50 \mu \mathrm{m}$ while the electron density adjacent to the surface is $2 \times 10^{14} \mathrm{~cm}^{-3}$ producing a Debye length of $0.7 \mu \mathrm{m}$. The resulting ion energies extend to as high as $30 \mathrm{eV}$ with an angular spread of $\pm 25^{\circ}$. At this time, the ion fluxes are $10^{17}-10^{18} \mathrm{~cm}^{-2} \mathrm{~s}^{-1}$.

The IEADs consist of two groups-an epithermal group that has a broad angular distribution and a high energy group with a narrow angular spread. The epithermal, broad energy portion of the IEAD is due to ions produced deep in the sheath by photoionization or those ions that have had collisions in the sheath. The high ion energy component of the IEAD is 


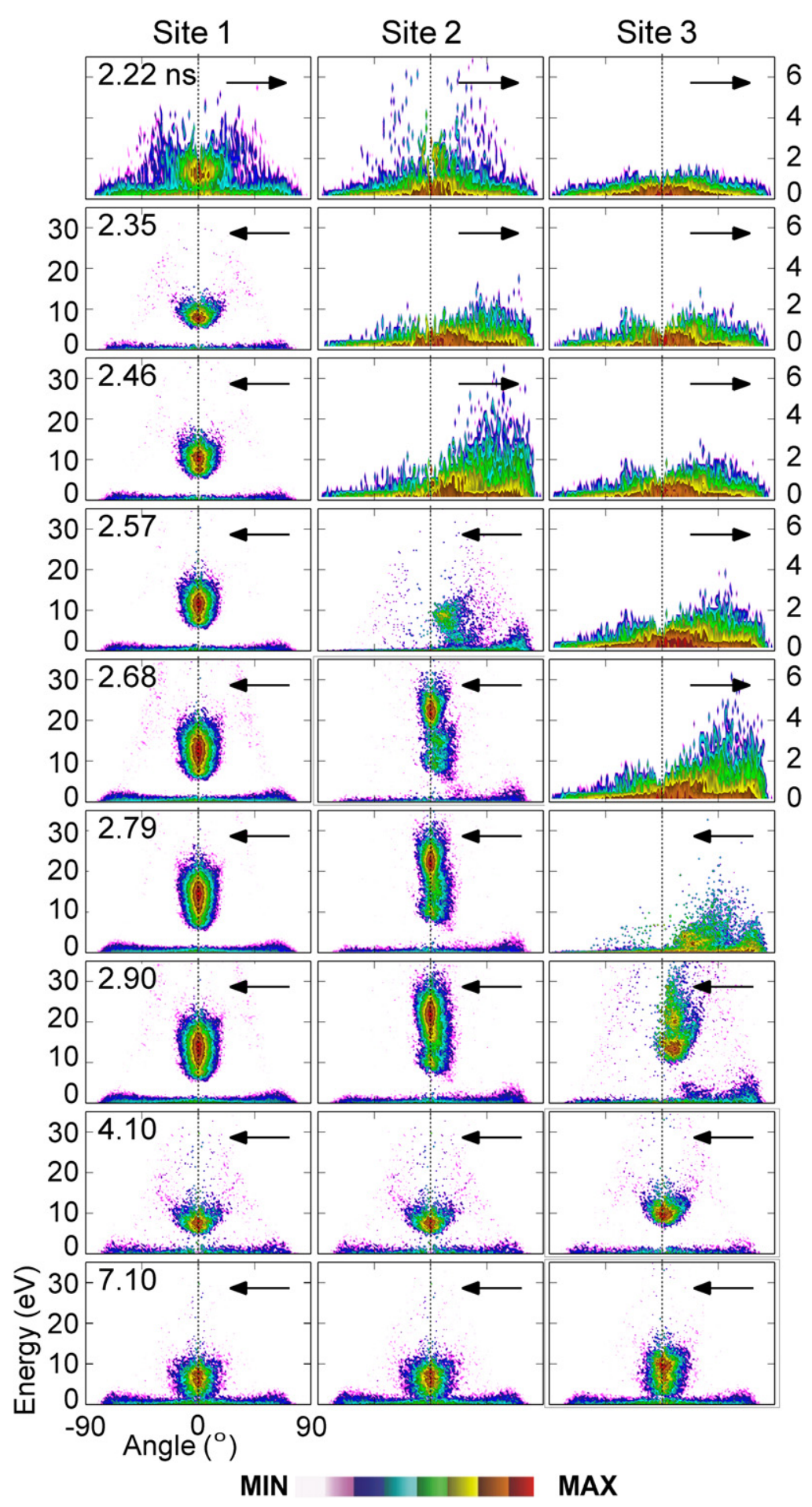

Figure 5. IEADs incident on the three sites along the polymer as a function of time corresponding to the filament positions shown in figure 2. Contours are plotted over 3 decades on a log-scale. The polymer and substrate have $\varepsilon / \varepsilon_{0}=2.2$ and the applied voltage is $15 \mathrm{kV}$. Ions are epithermal before the filaments approach the polymer. After the filament strikes the dielectric and the transient sheath is formed, two groups of ions are recorded-low energy ions and ions with energies as high as $25-30 \mathrm{eV}$. Site 1 is directly under the filament. There is a time delay for sites 2 and 3 as the filament spreads on the surface. The arrows in the frames indicate whether the right or left energy scale is used.

those ions that are largely formed in or near the sheath and are accelerated through the sheath from their point of generation with few collisions. The ion energy increases as the sheath voltage increases to a maximum value when the plasma channel is fully formed between the top dielectric and the polymer $(2.79 \mathrm{~ns})$. As the polymer charges and voltage is transferred to the underlying dielectric, the sheath voltage begins to decrease, reducing ion energies to $10 \mathrm{eV}$ at $7.1 \mathrm{~ns}$.

The IEADs for site 1 are essentially angularly symmetric as the streamer strikes the surface nearly vertically. As the streamer spreads along the surface, there are lateral components of the electric field produced in the direction of 
spreading. As a result, the IEADs striking the surface at sites 2 and 3 are produced by ions arriving asymmetrically skewed to positive angles. There is a time delay for production of the high energy component of the IEAD at site 2 and then at site 3 . This delay corresponds to the time required for the spreading of the discharge along the surface. (For a propagation speed of up to $10^{8} \mathrm{~cm} \mathrm{~s}^{-1}$, the delay between sites separated by $0.06 \mathrm{~cm}$ is $0.5 \mathrm{~ns}$.) The IEADs are most skewed in angle as the head of the streamer reaches the site. The parallel component of the electric field is largest as the streamer initially passes over the site (for example $2.57 \mathrm{~ns}$ for site 2 and $2.79 \mathrm{~ns}$ for site 3 ). After the surface discharge and sheath are formed atop the site, the electric fields are reoriented perpendicular to the surface, and the IEADS become angularly symmetric. This skewing and straightening of the IEADs then forms a wave that propagates across the surface. As the plasma decays with charging of the dielectric, ion energies gradually uniformly decrease with time for all sites.

Ion energy distributions (IEDs) integrated over angle are shown in figure 6 for different times. The wavelike production of the IEDs is shown by the similar IEDs at more remote sites with a time delay. Even for the highest compression of the potential and largest electric fields in the sheath $\left(400 \mathrm{kV} \mathrm{cm}^{-1}\right.$ at $2.79 \mathrm{~ns}$ at site 1 ) fewer than $10 \%$ of the ions exceed $10 \mathrm{eV}$ and only $1 \%$ of the ions exceed $20 \mathrm{eV}$. At site 3, a negligible fraction of ions $(0.3 \%)$ exceed $20 \mathrm{eV}$. However, similar to electron energy distributions, it is the ions that are above a given threshold energy that are important in activating a process.

Fluxes of $\mathrm{O}_{2}^{+}$and VUV photons striking the polymer are shown in figure 7 as a function of position and time. Ion fluxes increase with time as the streamer approaches the surface, from $10^{15} \mathrm{~cm}^{-2} \mathrm{~s}^{-1}$ to nearly $10^{19} \mathrm{~cm}^{-2} \mathrm{~s}^{-1}$ before saturating at $7 \mathrm{~ns}$. For a positive corona discharge, these fluxes represent the positive charging current to the substrate. The fluxes first rise at site 1 , directly under the streamer and subsequently rise on sites 2 and 3 as the discharge spreads on the surface. Although the currents remain high for many more ns due to the finite diffusion time of the ions, the sheath voltage has already significantly decayed by this time and so these ion fluxes are of lower energy ions. The VUV fluxes have values of up to $3 \times 10^{20} \mathrm{~cm}^{-2} \mathrm{~s}^{-1}$, similarly rising first on site 1 albeit for a short period. Photon fluxes increase up to $3 \mathrm{~ns}$ with a subsequent decrease due to the radiative relaxation of the originating states $\mathrm{N}_{2}^{* *}$. Once the avalanche front has passed over the site, the rate of excitation of high lying states decreases. Therefore, the $\mathrm{N}_{2}^{* *}$ states are not replenished after they radiate.

\subsection{High dielectric constant and voltage}

The IEADs striking the polymer are a function of its dielectric constant. Recall that the surface consists of a thin polymer layer (127 $\mu$ m thick, $\left.\varepsilon / \varepsilon_{0}=2.2\right)$ backed by a thicker dielectric whose dielectric constant can be varied. We chose this configuration with applications in mind. It is often the case that the $\varepsilon / \varepsilon_{0}$ of the material being processed in, for example, a corona discharge is invariant. If one is functionalizing the

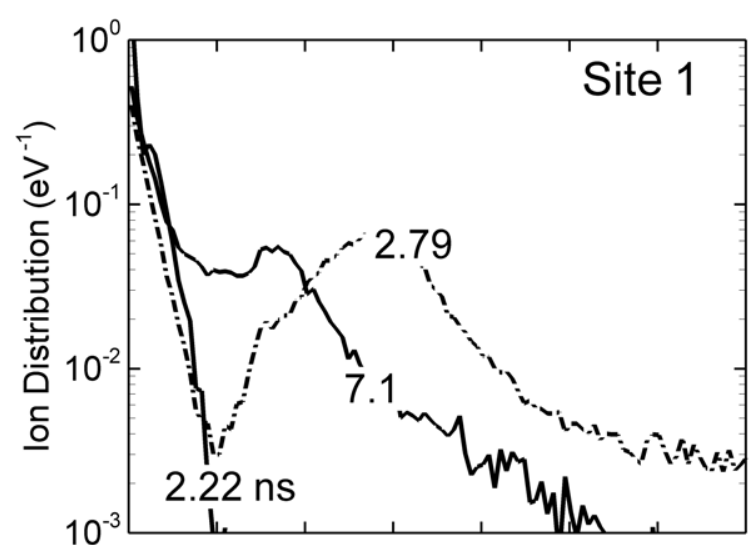

(a)

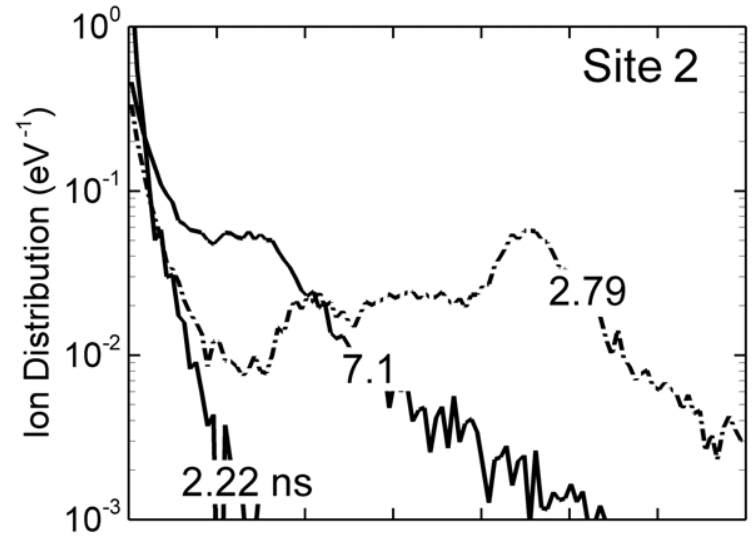

(b)

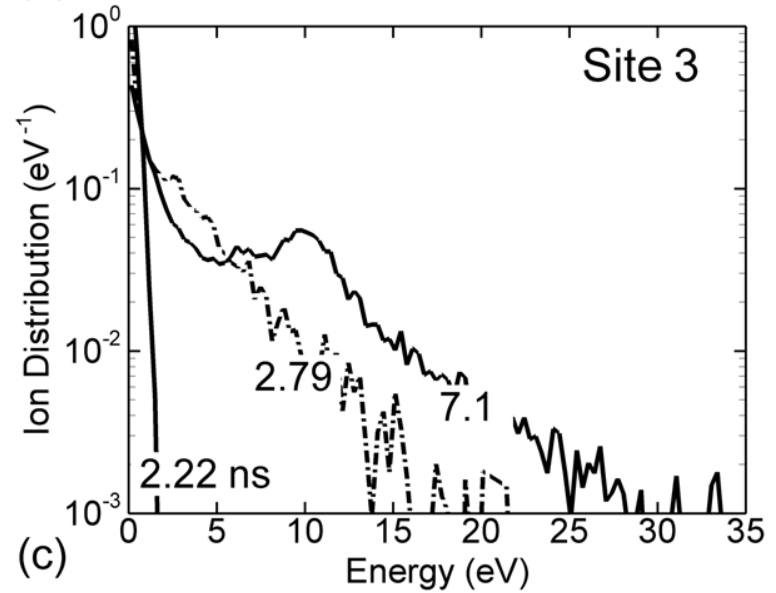

Figure 6. Ion energies at sites (a) 1, (b) 2 and (c) 3 integrated over angle during $0.1 \mathrm{~ns}$. The polymer and substrate have $\varepsilon / \varepsilon_{0}=2.2$ and the applied voltage is $15 \mathrm{kV}$. Prior to the filament striking the sites, ion energies are epithermal. After the transient sheath forms, ion energies as high as $25-30 \mathrm{eV}$ are recorded. The sheath decays with time resulting in a decrease in ion energies.

surface of a polypropylene sheet in a corona discharge, its dielectric constant cannot be changed as a process variable. However, the $\varepsilon / \varepsilon_{0}$ of the substrate under the material being processed can be more easily varied. Hence we investigated the consequences of varying the dielectric constant of the material underlying the polymer sheet.

When the streamer is far from the surface the differences in electric fields for different $\varepsilon / \varepsilon_{0}$ of the substrate are not particularly large. As the streamer approaches the surface, 


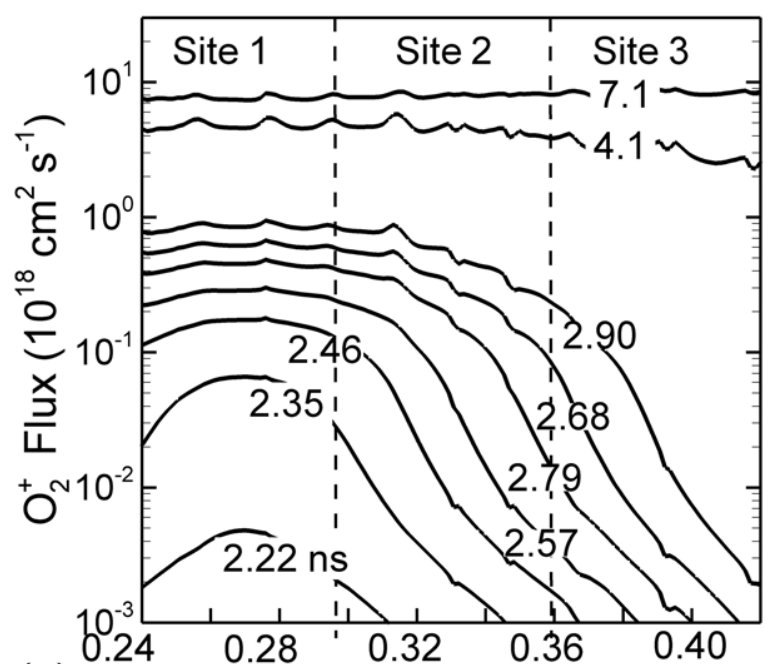

(a)

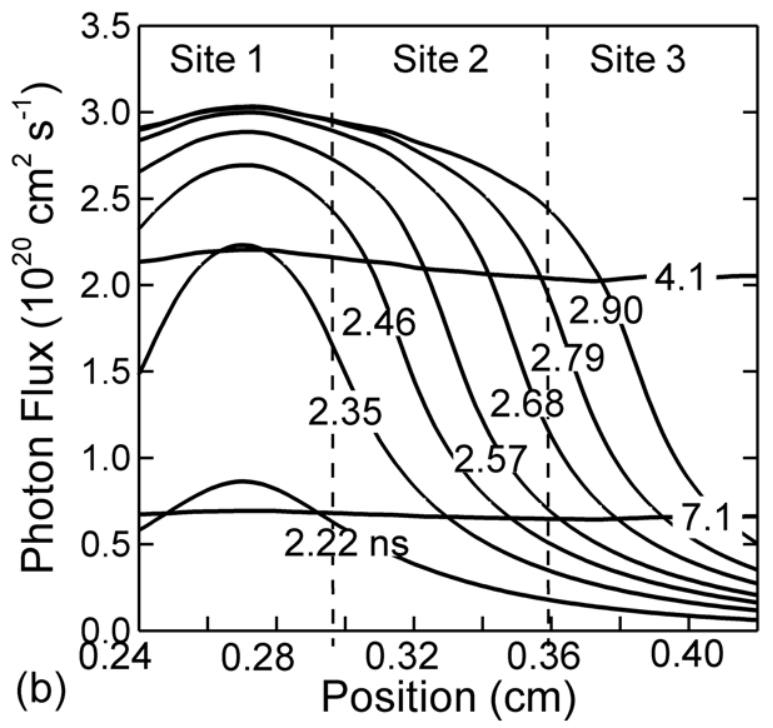

Figure 7. Fluxes of ( $a$ ) oxygen ions and $(b)$ photons arriving at sites 1,2 and 3 at different times. The polymer and substrate have $\varepsilon / \varepsilon_{0}=2.2$ and the applied voltage is $15 \mathrm{kV}$. Ion fluxes increase up to $7.1 \mathrm{~ns}$ while photon fluxes decrease beyond $3 \mathrm{~ns}$ due to the short lifetime of the emitting species.

voltage compression and transfer of potential to the sheath increase the electric field in the vicinity of the surface. The division of potential between the sheath, polymer sheet and underlying dielectric is determined by their relative capacitances. This is demonstrated in figure 8 where potential contours for substrates having different $\varepsilon / \varepsilon_{0}$ are shown at a time when the streamer strikes the dielectric. For small $\varepsilon / \varepsilon_{0}$, the capacitance more rapidly charges, thereby more rapidly removing voltage from the sheath. For large $\varepsilon / \varepsilon_{0}$, the capacitance is slow to charge, and so there is more voltage available for the sheath. Equivalently, with higher $\varepsilon / \varepsilon_{0}$, the potential lines are expelled from the substrate dielectric resulting in a higher voltage drop across the polymer and adjacent sheath.

The end result is that with substrates having large $\varepsilon / \varepsilon_{0}$, IEADs can extend to significantly higher energies. For example, electric fields as the plasma filament approaches and

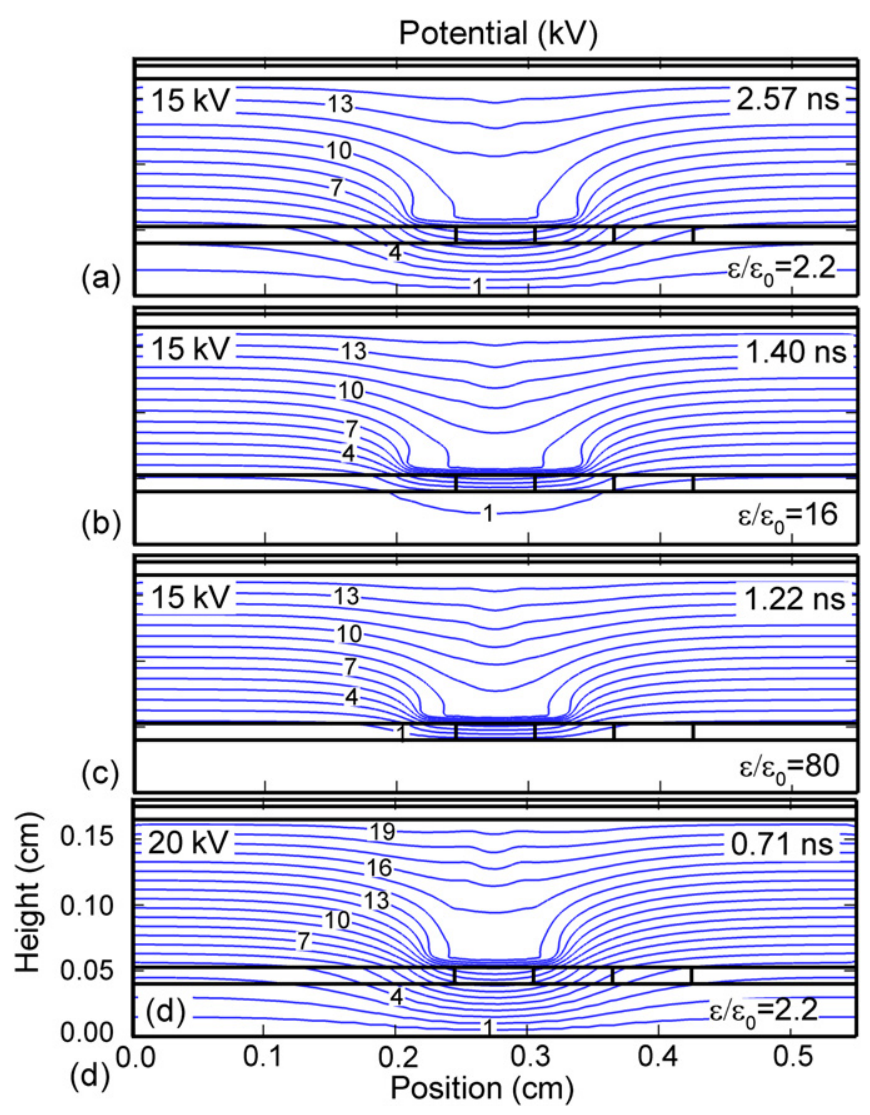

Figure 8. Potential contours for different substrate $\varepsilon / \varepsilon_{0}$ and applied voltage at the time when the streamer touches the polymer film. (a) Substrate $\varepsilon / \varepsilon_{0}=2.2, V=15 \mathrm{kV},(b)$ substrate $\varepsilon / \varepsilon_{0}=16$, $V=15 \mathrm{kV},(c)$ substrate $\varepsilon / \varepsilon_{0}=80, V=15 \mathrm{kV}$, and $(d)$ substrate $\varepsilon / \varepsilon_{0}=2.2, V=20 \mathrm{kV}$. With higher dielectric constant of the substrate, the potential lines are expelled from the dielectric resulting in a higher voltage drop across the polymer and adjacent sheath.

spreads over the surface and the resulting IEADs are shown in figure 9 for a substrate dielectric constant $\varepsilon / \varepsilon_{0}=16$. Electric fields and IEADs are shown in figure 10 for a substrate dielectric constant of $\varepsilon / \varepsilon_{0}=80$ (akin to that of water). The trends are similar to those for lower values of $\varepsilon / \varepsilon_{0}$ except that the maximum ion energies are higher, extending to $150 \mathrm{eV}$ for $\varepsilon / \varepsilon_{0}=16$ and $200 \mathrm{eV}$ for $\varepsilon / \varepsilon_{0}=80$. These larger ion energies are consistent with the larger electric fields in the sheath due to the slower charging of the dielectric. Due to this larger sheath voltage, the speed of spreading of the discharge along the surface increases with increasing $\varepsilon / \varepsilon_{0}$. For example, the arrival of the surface discharge at site 3 occurs at about $2.8 \mathrm{~ns}$ with $\varepsilon / \varepsilon_{0}=2.2,1.5 \mathrm{~ns}$ for $\varepsilon / \varepsilon_{0}=16$ and $1.3 \mathrm{~ns}$ for $\varepsilon / \varepsilon_{0}=80$.

Upon increasing $\varepsilon / \varepsilon_{0}$, the IEADs develop multiple peaks. These peaks are likely the result of symmetric charge-exchange collisions in which the resulting thermal ion is reaccelerated in the sheath electric field. Since the thermal ion results from charge transfer to another molecule of the same species, the symmetric charge-exchange reaction appears to be the transit of a single ion across the sheath that accelerates in the field and decelerates due to collisions. The higher the voltage drop in the sheath, and the greater the sheath thickness, the more likely that ions will be accelerated and decelerated multiple 

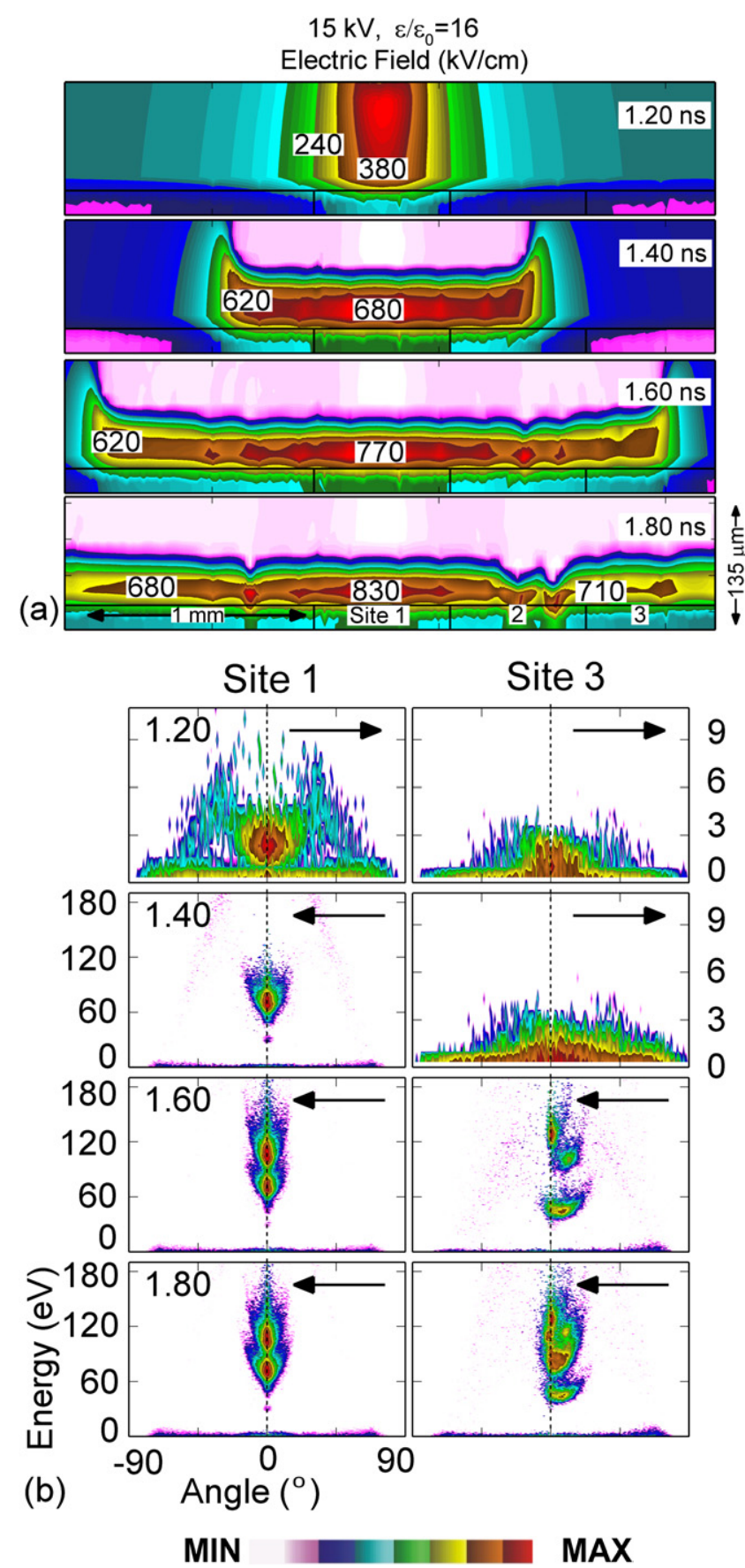

Figure 9. Plasma properties for substrate dielectric constant $\varepsilon / \varepsilon_{0}=16$ and applied voltage $15 \mathrm{kV}$. (a) Electric fields as the streamer approaches and spreads over the surface. (The vertical to horizontal scale ratio is 4$)$. (b) IEADs shown at the same times for sites 1 (symmetric distributions) and 3 (asymmetric distributions due to lateral electric fields). Contours are plotted over 3 decades on a log-scale. Ion energy increases up to $150 \mathrm{eV}$ due to partial expulsion of the potential from the substrate and more potential compression in the sheath region.

times across the sheath. Hence more peaks are observed in the IEADs. If the sheath were $\mathrm{dc}$ and the rate of influx of ions into the sheath or creation of ions in the sheath were continuous, one would not expect structure in the IEADs. The fact that we see such structure implies that the source of ions and the formation of the sheath are correlated. (a)
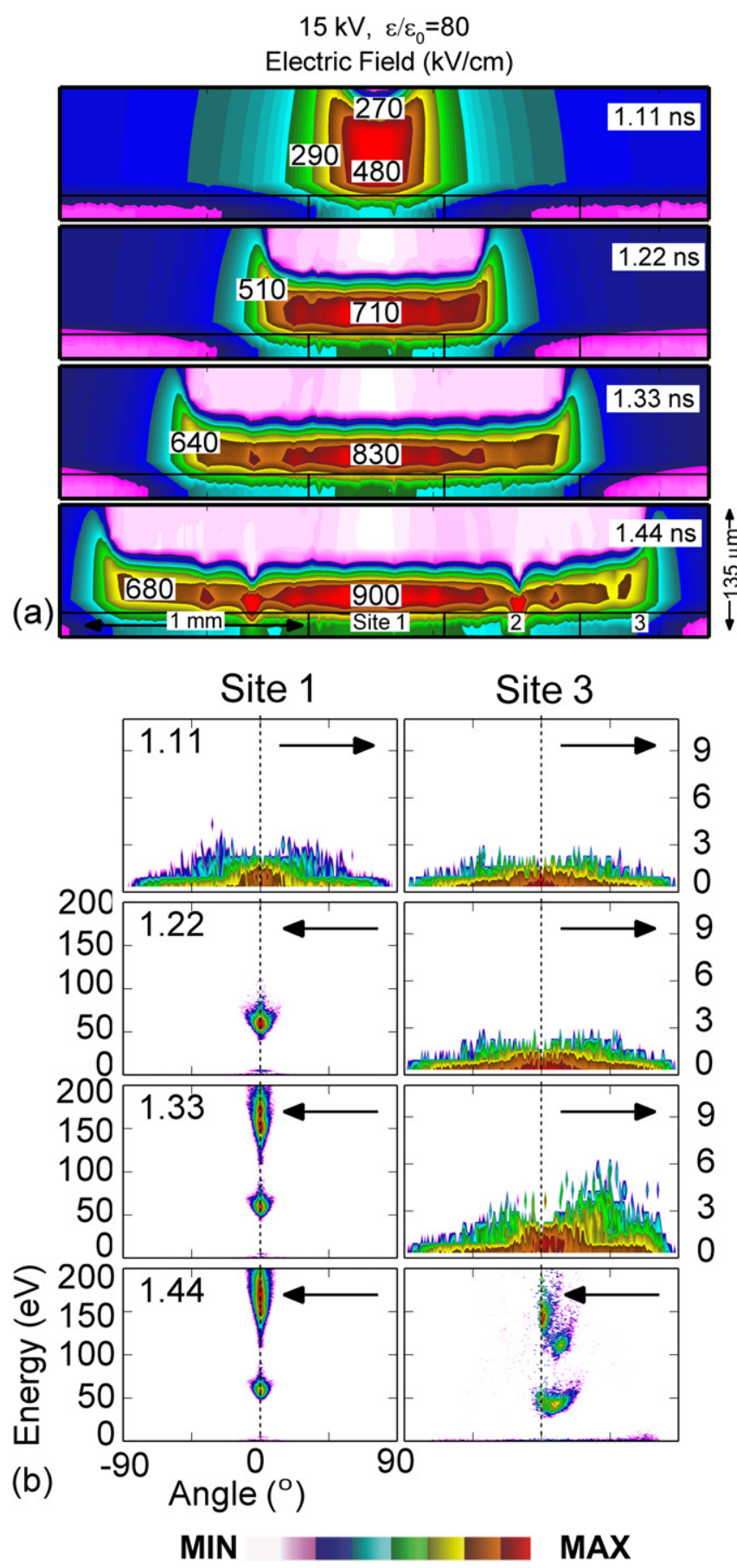

Figure 10. Plasma properties for substrate dielectric constant $\varepsilon / \varepsilon_{0}=80$ and applied voltage $15 \mathrm{kV}$. (a) Electric fields as the streamer approaches and spreads over the surface. (The vertical to horizontal scale ratio is 4). (b) IEADs shown at the same times for sites 1 (symmetric distributions) and 3 (asymmetric distributions due to lateral electric fields). Contours are plotted over 3 decades on a $\log$-scale. Ion energy increases up to $200 \mathrm{eV}$ due to the almost total expulsion of the potential from the substrate and more potential compression in the sheath region.

The trends in IEADs with increasing voltage are similar to those already discussed. The IEADs are simply shifted to higher energies. For example, electric fields as the filament approaches and spreads over the surface and the resulting IEADs are shown in figure 11 for a substrate having $\varepsilon / \varepsilon_{0}=2.2$ and applied voltage of $20 \mathrm{kV}$. Electric potential contours for 

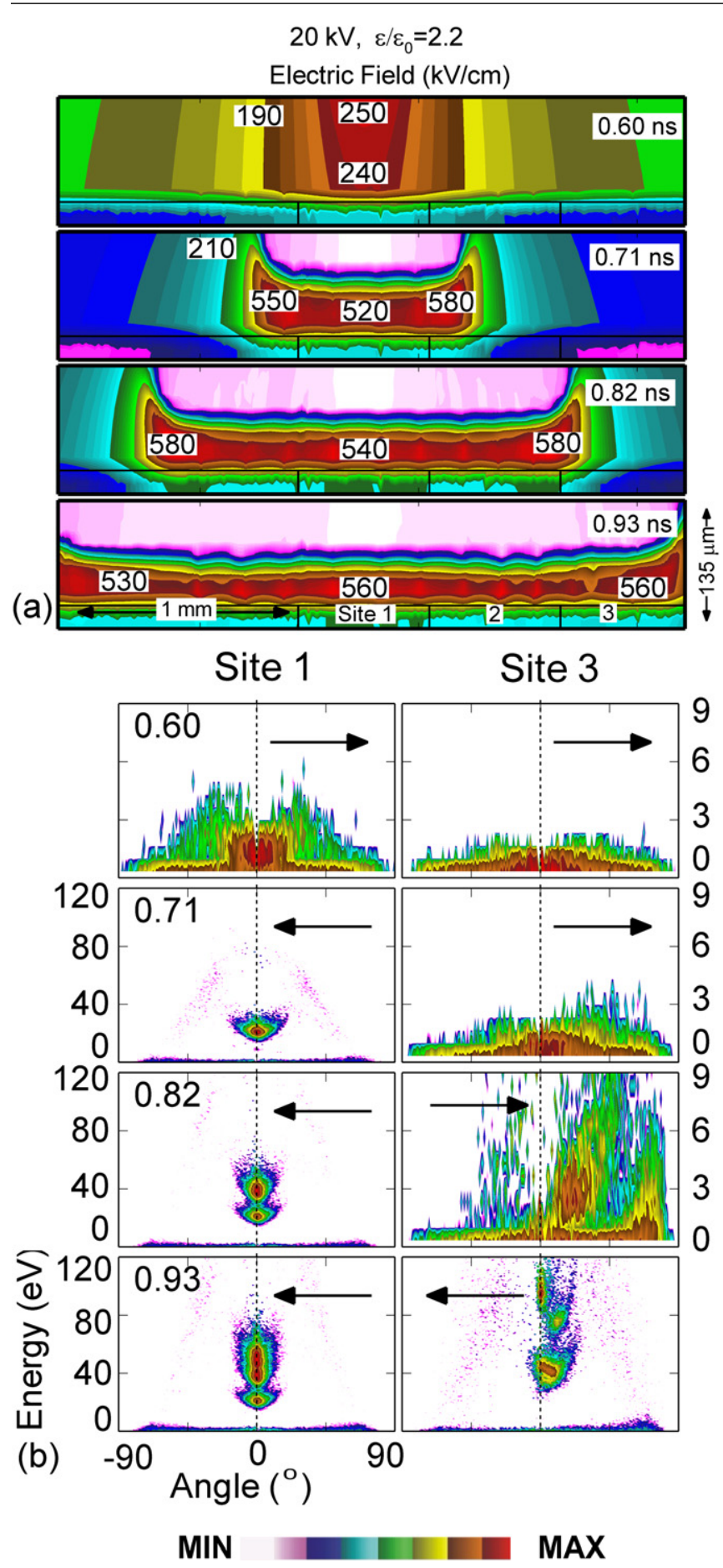

Figure 11. Plasma properties for substrate dielectric constant $\varepsilon / \varepsilon_{0}=2.2$ and applied voltage $20 \mathrm{kV}$. (a) Electric fields as the streamer approaches and spreads over the surface. (The vertical to horizontal scale ratio is 4$)$. (b) IEADs shown at the same times for sites 1 (symmetric distributions) and 3 (asymmetric distributions due to lateral electric fields). Contours are plotted over 3 decades on a log-scale. The trends are similar as at lower voltage except for there being higher ion energies and more asymmetries.

these conditions are in figure $8(d)$. The trends are similar to those described above with the exception of an increase in ion energy and a larger angular skew to the IEADs. This is likely a result of there being a greater voltage drop across the head of the discharge, and so there being larger lateral electric fields.

\section{Concluding remarks}

In plasma materials processing using DBDs at atmospheric pressure, it is typically assumed that the incident ions have near thermal energies. The results of this investigation suggest that ion energies may be significantly higher, though for short periods of time. With typical activation energies for chemical reactions of a few $\mathrm{eV}$ and sputtering thresholds of many to tens of $\mathrm{eV}$, these processes could be activated by ions having energies predicted here. Since the time over which there are significant fluxes of ions $\left(>10^{18}-10^{19} \mathrm{~cm}^{-2} \mathrm{~s}^{-1}\right)$ to the surface is only a few to tens of ns, and only a small fraction of the ions have above thermal energies, there is a limited amount of energy delivered to the surface in the form of energetic ions. For example, for streamer area densities of tens of $\mathrm{cm}^{-2}$ and repetition rates of $10 \mathrm{kHz}$, the power flux to the surface in the form of ions having energies greater than a few $\mathrm{eV}$ is about $1 \mathrm{~mW} \mathrm{~cm}^{-2}$. Hence the total energy expended in high energy ion bombardment of the surface is small. The issue then becomes whether this small flux of energetic ions can initiate reactions or processes that would not otherwise be produced by thermal ions or radicals.

Our investigation into IEADs may also be relevant to the field of plasma medicine wherein living tissue is treated with DBDs [9]. Depending on the applications, it may or may not be desirable to have ions of many tens of $\mathrm{eV}$ onto a surface depending on whether surface activation and bond breaking are desired. The dependence of the IEADs on underlying dielectrics is also important. In in vitro experiments, the IEADs incident on, for example, bacteria may depend on the $\varepsilon / \varepsilon_{0}$ of the underlying materials (i.e. growth medium, glass or plastic dish). Skin and wound treatment may depend on the wound conditions due to the permittivity of the surrounding medium. For example, mammalian skin consists of two main components: the epidermis with thickness of the order of $100 \mu \mathrm{m}$ and a thicker, collagenous, inner layer, the dermis. Wounded skin may have the dermis directly exposed. With the same applied voltage, ion energies onto these surfaces will likely be different dependent on the dielectric properties of the underlying tissues.

In conclusion, IEADs on polymer surfaces produced by atmospheric pressure DBD streamers were investigated. In the heads of streamers, electric fields are up to $200 \mathrm{kV} \mathrm{cm}^{-1}$ when the streamers are far from surfaces. When the streamer approaches dielectric surfaces, much of the electrode potential is compressed between the head and surface. Upon intersection of the streamer with flat polymer surfaces, the electric fields in the resulting sheath can exceed $400-800 \mathrm{kV} \mathrm{cm}^{-1}$. When accelerated in these high fields, ions can gain energies across their mean free path $(0.5-1 \mu \mathrm{m})$ of up to tens of $\mathrm{eV}$, approaching $100 \mathrm{eV}$ under extreme conditions. These energies are large enough for activating surface processes.

\section{Acknowledgment}

This work was supported by Department of Energy Office of Fusion Energy Sciences (DE-SC0001939, DE-SC0002588). 


\section{References}

[1] Strobel M, Lyons C S and Mittal K L (ed) 1994 Plasma Surface Modification of Polymers (Zeist, The Netherlands: VSP Press)

[2] Borcia G, Anderson C A and Brown N M D 2004 The surface oxidation of selected polymers using an atmospheric pressure air dielectric barrier discharge: I Appl. Surf. Sci. 221203

[3] Liu C, Cui N, Brown N M D and Meenan B J 2004 Effects of DBD plasma operating parameters on the polymer surface modification Surf. Coat. Technol. 185311

[4] Lopez-Santos C, Yubero F, Cotrino J, Barranco A and Gonzalez-Elipe A R 2008 Plasmas and atom beam activation of the surface of polymers J. Phys. D: Appl. Phys. 41225209

[5] Sira M, Trunec D, Stahel P, Bursikova V and Navratil Z 2008 Surface modification of polycarbonate in homogeneous atmospheric pressure discharge J. Phys. D: Appl. Phys. 41015205

[6] Fang Z, Xie X, Li J, Yang H, Qiu Y and Kuffel E 2009 Comparison of surface modification of polypropylene film by filamentary DBD at atmospheric pressure and homogeneous DBD at medium pressure in air J. Phys. D: Appl. Phys. 42085204

[7] Papageorghiou L, Panousis E, Loiseau J F, Spyrou N and Held B 2009 Two-dimensional modeling of a nitrogen dielectric barrier discharge (DBD) at atmospheric pressure: filament dynamics with the dielectric barrier on the cathode J. Phys. D: Appl. Phys. 42105201

[8] Kong M G, Kroesen G, Morfill G, Nosenko T, Shimizu T, van Dijk J and Zimmermann J L 2009 Plasma medicine: an introductory review New J. Phys. 11115012

[9] Fridman G, Peddinghaus M, Ayan H, Fridman A Balasubramanian M, Gutsol A, Brooks A and Friedman G 2006 Blood coagulation and living tissue sterilization by floating-electrode dielectric barrier discharge in air Plasma Chem. Plasma Process. 26 425-42

[10] Kulikovsky A A 1998 Positive streamer in a weak field in air: a moving avalanche-to-streamer transition Phys. Rev. E 577066

[11] Babaeva N Yu and Kushner M J 2011 Ion energy and angular distributions onto polymer surfaces delivered by dielectric barrier discharge filaments in air: II. Particles Plasma Sources Sci. Technol. 20035018

[12] Babaeva N Yu and Kushner M J 2009 Effect of inhomogeneities on streamer propagation: I. Intersection with isolated bubbles and particles Plasma Sources Sci. Technol. 18035009

[13] Agarwal A and Kushner M J 2005 Effect of nonsinusoidal bias waveforms on ion energy distributions and fluorocarbon plasma etch selectivity J. Vac. Sci. Technol. A 231440

[14] Celestin S, Canes-Boussard G, Guaitella O, Bourdon A and Rousseau A 2008 Influence of the charges deposition on the spatio-temporal self-organization of streamers in a DBD $J$. Phys. D: Appl. Phys. 41205214

[15] Stollenwerk L 2009 Interaction of current filaments in dielectric barrier discharges with relation to surface charge distributions New J. Phys. 11103034

[16] Celestin S, Bonaventura Z, Guaitella O, Rousseau A and Bourdon A 2009 Influence of surface charges on the structure of a dielectric barrier discharge in air at atmospheric pressure: experiment and modeling Eur. Phys. J. Appl. Phys. 4722810

[17] Starikovskaia S M, Allegraud K, Guaitella O and Rousseau A 2010 On electric field measurements in surface dielectric barrier discharge J. Phys. D: Appl. Phys. 43124007

[18] Mason E A and McDaniel E W 1988 Transport Properties of Ions in Gases (New York: Wiley) 